\title{
Evidence-Based Multidisciplinary Design Optimization with the Active Global Kriging Model
}

\author{
Fan Yang $\mathbb{( D},{ }^{1,2,3}$ Ming Liu $\mathbb{D},{ }^{3}$ Lei Li $\left(\mathbb{D},{ }^{4}\right.$ Hu Ren $\left(\mathbb{D},{ }^{5}\right.$ and Jianbo Wu $\mathbb{D}^{5}$ \\ ${ }^{1}$ State Key Laboratory of Mechanics and Control of Mechanical Structures, Nanjing University of Aeronautics and Astronautics, \\ Nanjing 210016, China \\ ${ }^{2}$ College of Aerospace Engineering, Nanjing University of Aeronautics and Astronautics, Nanjing 210016, China \\ ${ }^{3}$ State Key Laboratory for Strength and Vibration of Mechanical Structures, Xi'an Jiaotong University, Xi'an 710049, China \\ ${ }^{4}$ Department of Engineering Mechanics, Northwestern Polytechnincal University, Xi'an 710072, China \\ ${ }^{5}$ Wuxi Hengding Supercomputing Center Ltd., Wuxi 214135, China
}

Correspondence should be addressed to Fan Yang; fanyang@nuaa.edu.cn

Received 25 March 2019; Revised 11 September 2019; Accepted 26 September 2019; Published 15 November 2019

Academic Editor: Eulalia Martínez

Copyright (c) 2019 Fan Yang et al. This is an open access article distributed under the Creative Commons Attribution License, which permits unrestricted use, distribution, and reproduction in any medium, provided the original work is properly cited.

This article presents an approach that combines the active global Kriging method and multidisciplinary strategy to investigate the problem of evidence-based multidisciplinary design optimization. The global Kriging model is constructed by introducing a socalled learning function and using actively selected samples in the entire optimization space. With the Kriging model, the plausibility, Pl, of failure is obtained with evidence theory. The multidisciplinary feasible and collaborative optimization strategies of multidisciplinary design optimization are combined with the evidence-based reliability analysis. Numerical examples are provided to illustrate the efficiency and accuracy of the proposed method. The numerical results show that the proposed algorithm is effective and valuable, which is valuable in engineering application.

\section{Introduction}

The performance of structures in engineering system is often influenced by uncertainties. The optimal solution obtained by conventional deterministic design frequently falls on the boundary of the feasible region [1]. The deterministic design may fall into the failure region if the system is disturbed by uncertainties. In the multidisciplinary coupling systems, the propagation of uncertainty factors between different subjects makes the search for an optimal design more complicated as well as difficult than the search for an optimal individual discipline design due to the coupling effect $[2,3]$. The influence of uncertainty factors must be considered to ensure the reliability of the optimized design results, and reliabilitybased multidisciplinary design optimization (RBMDO) needs to be performed $[4,5]$.

Uncertainties can be categorized as aleatory and epistemic $[6,7]$. Aleatory or objective uncertainties arise from the inherent randomness of a system. Probability theory can be adopted to handle random uncertainties. However, sufficient information is required to construct the distribution function of uncertain variables. Epistemic or subjective uncertainties stem from the lack of sufficient information during modeling and optimization. Several theories, including probability box models, fuzzy sets, Bayesian approaches, and evidence theory, have been developed to deal with epistemic uncertainties [8-15]. Evidence theory provides a general modeling of epistemic uncertainty and can be reduced to other theories. When the interval of evidence theory is infinite, it is equivalent to traditional probability theory. When no conflict exists between uncertain pieces of information, the theory is equivalent to possibility theory. When the subinterval of the evidence variable is unique, the theory degenerates into convex model theory.

Several methods have been proposed for reliability analyses based on evidence theory, and these approaches include Cartesian product method (CPM) [16], Monte Carlo 
simulation (MCS) [17-20], polynomial chaos expansion [21], and surrogate-based models [22-24]. Design optimization based on evidence theory has also been studied [25-28]. Zhang et al. [29] adopted two-stage framework to handle the evidence-based design optimization problem, where the evidence variables were transformed into random variables and the sequential optimization and reliability assessment were used. $\mathrm{He}$ and $\mathrm{Qu}[30]$ gave an overview of possibility and evidence theory-based design optimization. However, limited research has been conducted on evidence-based multidisciplinary design optimization (EBMDO) [31-33]. Conventional EBMDO has a three-level loop architecture. The outer loop is multidisciplinary optimization, the middle loop is reliability analysis based on evidence theory, and the inner loop is discipline analysis. The computational cost of EBMDO is high, especially in the inner discipline analysis. Practical engineering problems require repeatedly calling the simulation model to evaluate limit state and objective functions, and this process is time consuming.

To address this issue, this study proposes a surrogate model-based method with an active global learning strategy, which constructs surrogate models in entire design space with intelligent sampling approach, to reduce the amount of computation [34]. The active learning Kriging [35] model is utilized as a surrogate model to evaluate the limit state function in evidence-based reliability analysis. Since the original active Kriging model is utilized for reliability analysis, a model in local region, here the Kriging model is constructed in the entire design space called active global Kriging model. Then, two multidisciplinary integration frameworks, namely, Multidisciplinary Feasible (MDF) and Collaborative Optimization (CO), are adopted and combined with the Kriging model. Using the global Kriging model, the computational cost of EBMDO is reduced to the approximate equivalent in deterministic multidisciplinary design optimization (MDO).

The rest of the article is organized as follows. MDF and CO strategies for EBMDO are briefly introduced in Section 2. An evidence-based reliability analysis using the global kriging model for MDO is presented in detail in Section 3. Numerical examples are provided and discussed in Section 4, and the conclusions are presented in Section 5.

\section{Methodology}

2.1. EBMDO. The general EBMDO model is expressed as

$$
\begin{array}{ll}
\text { find } & \left\{\mathbf{d}_{s}, \mathbf{d}, \mu_{\mathbf{X}_{s}}, \mu_{\mathbf{X}}\right\}, \\
\min & f\left(\mathbf{d}_{s}, \mathbf{d}, \mu_{\mathbf{X}_{s}}, \mu_{\mathbf{X}}, \mu_{\mathbf{K}}\right), \\
\text { s.t. } & \operatorname{Pl}\left[G_{i}\left(\mathbf{d}_{s}, \mathbf{d}_{i}, \mathbf{X}_{s}, \mathbf{X}_{i}, \mathbf{K}_{\bullet}\right)<0\right]<1-\Phi(\beta), \quad i=1, \ldots, n,
\end{array}
$$

where $\mathbf{d}$ pertains to the deterministic variables, $\mathbf{d}_{s}$ pertains to shared design variables that are common among all disciplines, $\mathbf{d}_{i}$ pertains to the design variables of the $i^{\text {th }}$ discipline, $\mathbf{X}$ pertains to the uncertain variables, $\mu_{X}$ denotes the mean of the $X$, which is treated as the uncertain design variables, $\mathbf{X}_{s}$ pertains to uncertain shared evidence variables that are the common input variables for all disciplines, and $\mathbf{X}_{i}$ pertains to the local evidence variables of the $i^{\text {th }}$ discipline. The shared variable $\mathbf{X}_{s}$ and the input variable $\mathbf{X}$ are independent variables, $\mathbf{K}$ encompasses coupling variables, and $f$ is the objective function. $\mathrm{Pl}[\cdot]$ is the upper bound of failure probability which represents the worst-case and is called plausibility. $\operatorname{Pl}\left[G_{i}(\cdot)<0\right]<1-\Phi(\beta)$ is the failure probability constraint of the $i^{\text {th }}$ discipline, and $G_{i}(\cdot)$ is the limit state of the $i^{\text {th }}$ discipline. The failure model is defined by $G_{i}(\cdot)<0$. $\beta$ is the reliability index, and $\Phi(\cdot)$ denotes the cumulative distribution function (CDF) of the standard normal distribution. The coupling variables between design and uncertain variables are expressed as

$$
\mathbf{K}_{\bullet i}=\mathbf{K}_{j i}\left(\mathbf{d}_{s}, \mathbf{d}_{i}, \mathbf{X}_{s}, \mathbf{X}_{i}\right), \quad j=1, \ldots, n, j \neq i,
$$

where the first subscript $j$ and the second subscript $i$ of $\mathbf{K}_{j i}$ denote the values from the $j^{\text {th }}$ discipline to the $i^{\text {th }}$ discipline. The meaning of $\mathbf{K}_{j i}$ can be explained in detail that the coupling variables are inner shared variables in the $j^{\text {th }}$ discipline and the $i^{\text {th }}$ discipline, and $\mathbf{K}_{j i}$ is the output values of the $j^{\text {th }}$ discipline and the input values for the $i^{\text {th }}$ discipline. For example, in fluid structure thermal coupling analysis, the pressure values from fluid analysis should be transferred to the structure discipline for finite element analysis. The conventional structure of EBMDO contains a triple loop, as shown in Figure 1 [36, 37].

As shown in Figure 1, due to the nested architecture, failure plausibility must be calculated at every design point during optimization. Therefore, the multidisciplinary analysis is repeatedly called to evaluate the limit state function. Furthermore, according to evidence theory, the optimization problem should be implemented to determine the upper bound of the limit state. This nested structure makes EBMDO costly.

2.2. Multidisciplinary Feasible Method. The framework of MDO aims to manage coupled variables and subsystems and improve calculation efficiency. The MDO framework has two main forms, namely, single-level and multilevel structures.

MDF is a traditional single-level optimization method. A systematic analysis is performed for each iteration during optimization, and a multidisciplinary analysis is performed repeatedly to obtain consistent solutions of the coupling variables among disciplines. This optimization framework includes a system of integrated and subsystem analysis modules. Only one interface exists for the input and output between the system module and subsystem modules, as shown in Figure 2. The system module is an optimizer to search for the optimal solution. The subsystem modules are for multidisciplinary and reliability analyses. In each optimization iteration, the coupling variables should be consistent and the constraints should be also satisfied. Therefore, when the optimal solution is obtained, the consistency constraints of coupling variables and reliability constraints are satisfied.

2.3. Collaborative Optimization Method. CO is a bilevel framework of MDO. The framework of MDO is designed to 


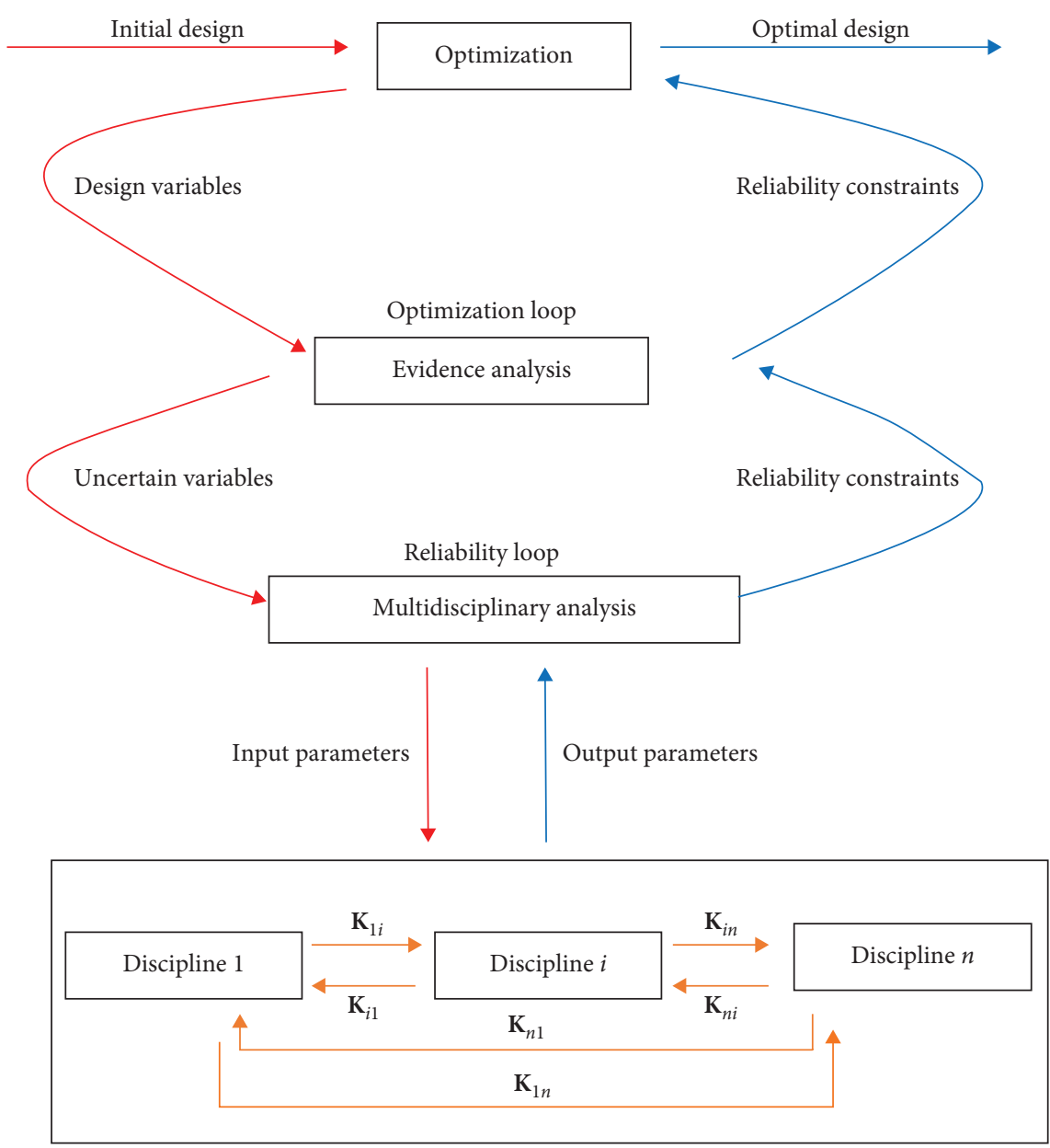

FIgURE 1: Conventional procedure of RBMDO.

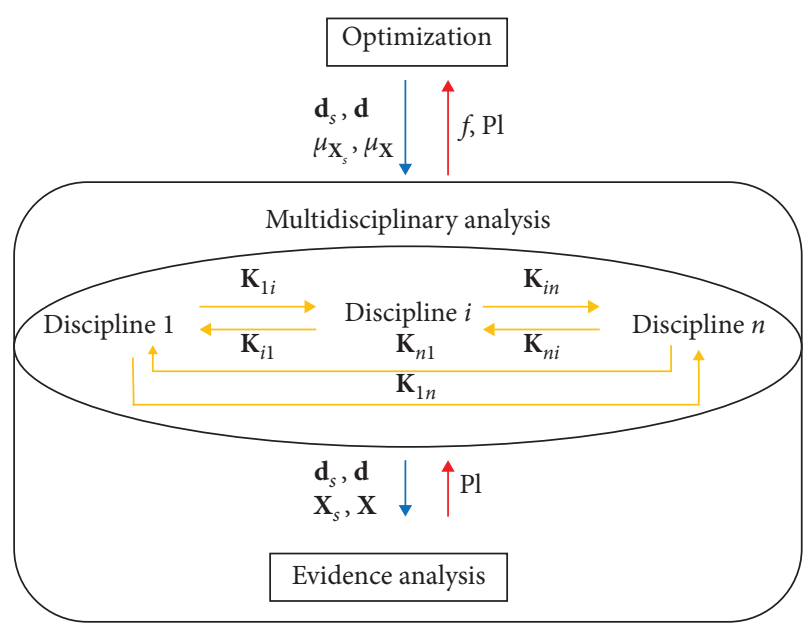

FIgURE 2: MDF architecture of EBMDO.

be top level (system level) and low level (discipline level) by CO. The top level is a system optimizer responsible for assigning target values for system-level state variables to disciplines. The low level is a parallel, distributed, multiplediscipline subsystem. In addition to satisfying the constraints of the subsystems, the objective function is the smallest difference between the coupling state variables of the top-level and low-level systems. After low-level system optimization, the objective function is fed back to the toplevel system, which constitutes the top-level consistency constraint. Optimization of the top-level system is performed to address the uncoordinated state variables among the subsystems. The framework for $\mathrm{CO}$ is defined as follows:

$$
\begin{aligned}
\min & f\left(\mathbf{d}_{s_{-} \mathrm{s}}, \mathbf{d}_{\mathrm{sl}}, \mu_{\mathbf{X}_{s_{-} \mathrm{s}}}, \ldots, \mu_{\mathbf{X}_{\mathrm{sl}}}, \mu_{\mathbf{P}_{\mathrm{sl}}}, \mu_{\mathrm{K}_{\mathrm{sl}}}\right), \\
\text { s.t. } & J_{i}^{*}=0, \quad i=1, \ldots, N, \\
& \mathbf{d}_{s}^{L} \leq \mathbf{d}_{s_{-} \mathrm{sl}} \leq \mathbf{d}_{s}^{U}, \\
& \mathbf{d}^{L} \leq \mathbf{d} \leq \mathbf{d}^{U}, \\
& \mathbf{X}_{s}^{L} \leq \mu_{\mathbf{X}_{s_{-s} \mathrm{sl}}} \leq \mathbf{X}_{s}^{U}, \\
& \mathbf{X}^{L} \leq \mu_{\mathbf{X}} \leq \mathbf{X}^{U},
\end{aligned}
$$

where $f$ is the objective function of system-level problems, $\mathbf{d}_{s_{-} s l}$ denotes the shared deterministic design variables of system-level problems, $\mathbf{d}_{\mathrm{sl}}$ denotes the deterministic design variables of discipline-level problems, $\mathbf{X}_{s_{-} s l}$ denotes the shared uncertain design variables of system-level problems, $\mathbf{X}_{\mathrm{sl}}$ denotes the uncertain design variables of the discipline- 
level problems, $\mathbf{P}_{\mathrm{sl}}$ denotes the global uncertain parameters, and $J^{*}$ is the constraint of the discipline-level problems.

The $i^{\text {th }}$ discipline subproblem is

$$
\begin{array}{ll}
\min & J_{i}=\left\|\mathbf{d}_{s_{-} \text {sl }}-\mathbf{d}_{s i}\right\|^{2}+\left\|\mathbf{d}_{\mathrm{sl}}-\mathbf{d}_{i}\right\|^{2}+\left\|\mu_{\mathbf{X}_{s_{-} s \mathrm{~s}}}-\mu_{\mathbf{X}_{s i}}\right\|^{2} \\
& +\left\|\mu_{\mathbf{X}_{\mathrm{sl}}}-\mu_{\mathbf{X}_{i}}\right\|^{2}, \\
& \text { s.t. } \quad \operatorname{Pl}_{i j}\left(g_{i j}\left(\mathbf{X}_{s}, \mathbf{X}, \mathbf{P}\right)<0\right)<1-\Phi(\beta), j=1, \ldots, m, \\
& \mathbf{X}^{L} \leq \mu_{\mathbf{X}} \leq \mathbf{X}^{U},
\end{array}
$$

where $J_{i}$ is the objective of the discipline-level problems, $\mathbf{d}_{\mathrm{sl}}$ denotes the shared design variables, $\mathbf{d}_{i}$ denotes the design variables of the $i^{\text {th }}$ discipline, ${ }_{s i}$ denotes the shared uncertain design variables, and $\mathbf{X}_{i}$ denotes the uncertain design variables of the $i^{\text {th }}$ discipline.

The diagram of CO for EBMDO is shown in Figure 3.

\section{Reliability Analysis}

3.1. Reliability Analysis Based on Evidence Theory. The limit state function is denoted as $G(x)$. Failure domain $F$ is defined as $F=\{G: G(x)<0\}$, where the evidence variables are $x=\left[x_{1}, x_{2}, \ldots, x_{n X}\right]$. The evidence variable $x_{j}$ can be described by evidence space $\left(X_{E j}, m_{E j}\right)$, where $X_{E j}$ is the set of all focal elements [21] and $X_{E j}=\left\{A_{j}^{1}, A_{j}^{2}, \ldots, A_{j}^{n_{j}}\right\}$, where $A_{j}^{i}$ is the $i^{\text {th }}$ focal element. In the evidence analysis, the focal element is usually an interval. $m_{E j}$ is the basic probability assignment (BPA) of each focal element. The joint focal element (JFE) is defined by CPM:

$$
A_{k}=\left\{A_{1}, A_{2}, \ldots, A_{n_{E}} \mid A_{k}=x_{j=1}^{n_{X}} A_{j}^{(i)}, A_{j}^{(i)} \in A_{j}\right\} .
$$

The BPA associated with JFE is calculated by

$$
m_{E}\left(A_{k}\right)=\prod_{j=1}^{n_{X}} m_{E j}\left(A_{j}^{(i)}\right),
$$

where $n_{X}$ is the number of JFEs. In the evidence space, failure probability $P_{\mathrm{f}}$ is an interval. The lower and upper bounds of the interval are called belief (Bel) and plausibility $(\mathrm{Pl})$, respectively. Bel means that the system is in the absolute failure state, and $\mathrm{Pl}$ means that the system is in the possible failure state. The belief and plausibility measures of failure probability are calculated by

$$
\begin{aligned}
\operatorname{Bel}(F) & =\sum_{k=1}^{n_{E}} I\left(A_{k} \subseteq F\right) m\left(A_{k}\right), \\
\operatorname{Pl}(F) & =\sum_{k=1}^{n_{E}} I\left(A_{k} \cap F \neq \varnothing\right) m\left(A_{k}\right),
\end{aligned}
$$

where $I(\cdot)$ is the indicator function.

$$
I(\cdot)= \begin{cases}1, & \text { true } \\ 0, & \text { false }\end{cases}
$$

The equivalent form of equations (7) and (8) are

$$
\begin{aligned}
\operatorname{Bel}(F) & =\sum_{k=1}^{n_{E}} I\left(\max _{x \in A_{k}} G(x)<0\right) m\left(A_{k}\right), \\
\operatorname{Pl}(F) & =\sum_{k=1}^{n_{E}} I\left(\min _{x \in A_{k}} G(x)<0\right) m\left(A_{k}\right) .
\end{aligned}
$$

This method involves a combinatorial explosion problem because it needs to explore the extrema of $G(x)$ in $n_{E}$ JFEs. According to equations (7) and (8), if $n_{1}$ variables and $n_{2}$ focal elements exist for each variable, then the total number of JFEs will be $n_{2}^{n_{1}}$. The Monte Carlo method is adopted to randomly sample the focal elements and overcome the obstacle of dimensions. JFEs are formed by these focal elements. An extremum analysis is performed based on JFEs, and Bel and $\mathrm{Pl}$ can be estimated using statistical methods, shown as

$$
\begin{aligned}
& \operatorname{Bel}(F) \frac{1}{N} \sum_{k=1}^{N} I\left(\max _{x \in A_{k}} G(x)<0\right), \\
& \operatorname{Pl}(F)=\frac{1}{N} \sum_{k=1}^{N} I\left(\min _{x \in A_{k}} G(x)<0\right),
\end{aligned}
$$

where $N$ is the number of samples for the Monte Carlo simulation (MCS).

3.2. Evidence Analysis with the Kriging Model. Although the dimensionality challenge is addressed, the computational cost remains large for MCS. To further improve the computational efficiency, the Kriging model is proposed to evaluate the limit state function in a multidisciplinary coupling system. The Kriging model is briefly introduced as follows.

Suppose that a set of $N$ samples called design of experiments (DoE) has the form $(\mathbf{x}, G)$, where $\mathbf{x}$ is an $n$-dimensional vector $\left[x^{(1)}, x^{(2)}, \ldots, x^{(n)}\right]^{T}$ and $G$ is the corresponding output $\left[G\left(x^{(1)}\right), G\left(x^{(2)}\right), \ldots, G\left(x^{(n)}\right)\right]^{T}$. The predicted value $G(\mathbf{x})$ and variance $s^{2}(\mathbf{x})$ are given as

$$
\begin{aligned}
& G(\mathbf{x})=\boldsymbol{\beta}+\mathbf{r}(\mathbf{x})^{T} \mathbf{R}^{-1}(G-\boldsymbol{\beta}), \\
& s^{2}(\mathbf{x})=\sigma^{2}\left[1+\frac{\left(1^{T} \mathbf{R}^{-1} \mathbf{r}-1\right)^{2}}{1^{T} \mathbf{R}^{-1} 1}-\mathbf{r}^{T} \mathbf{R}^{-1} \mathbf{r}\right],
\end{aligned}
$$

where $\mathbf{1}$ is an $n$-dimensional unit vector, $\mathbf{r}$ is the vector of the correlation function of $\mathbf{x}$ and points in DoE, $\mathbf{R}$ is the matrix of the correlation function of points in DoE, and $\beta, \sigma^{2}$ are the parameters to be determined. The methods of the fitting parameters are adopted as modified DIRECT algorithm [38]. Further details about the Kriging model can be found in Ref. [39].

For evidence-based design optimization, the Kriging model is used to approximate the limit state function in the entire design space. The idea here is that if the sign given by the Kriging model is the same to that of the true limit state function, then the result of MCS combined with the Kriging model can be considered trustworthy. The following 


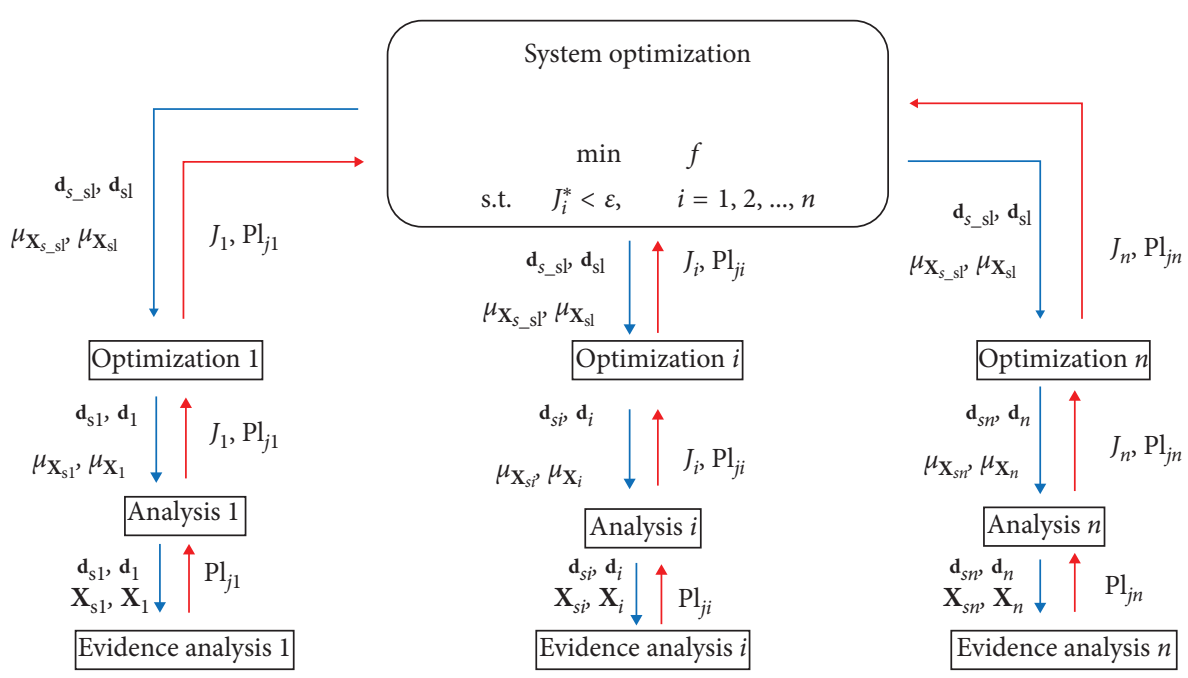

FIgURE 3: CO architecture of EBMDO.

property can ensure that the sign of the extremum value by the Kriging model is consistent with that of the true limit state function.

Property. In the entire design space $\Omega$, if $\operatorname{sign}(\widehat{G}(X))=$ $\operatorname{sign}(G(X))$, then in the local design space $\Omega_{\text {local }}$, $\operatorname{sign}(\min (\widehat{G}(X)))=\operatorname{sign}(\min (G(X)))$ and $\operatorname{sign}(\max$ $(\widehat{G}(X)))=\operatorname{sign}(\max (G(X)))$ where $(\operatorname{sign}(\cdot))$ is the sign function.

Proof. Consider the case of the minimum. Suppose $X_{\min }^{*}$ is the minimum point of $\widehat{G}(X)$, and $X_{\min }^{*}$ is the minimum point of $G(X)$, namely, $\widehat{G}\left(X_{\min }^{*}\right)=\min (\widehat{G}(X))$ and $G\left(X_{\min }^{*}\right)=\min ((X))$. We will discuss in two cases.

Case 1. If $\widehat{G}\left(X_{\min }^{*}\right)<0$ because sign $(\widehat{G}(X))=\operatorname{sign}(G(X))$, then $G\left(X_{\min }^{*}\right)<0$ because $G\left(X_{\min }^{*}\right)=\min (G(X))$, so $G\left(X_{\min }^{*}\right) \leq G\left(X_{\min }^{*}\right)<0$.

Case 2. If $\widehat{G}\left(X_{\min }^{*}\right)>0$ because $\widehat{G}\left(X_{\min }^{*}\right)=\min (\widehat{G}(X))$, then $0<\widehat{G}\left(X_{\min }^{*}\right) \leq \widehat{G}\left(X_{\min }^{*}\right)$ because sign $(\widehat{G}(X))=\operatorname{sign}(G(X))$, so $G\left(X_{\min }^{*}\right)>0$.

Therefore, when the limit state function has a minimum value, the sign by the Kriging model and the true limit state function are the same. The case of the maximum value can be proven in a similar way. Suppose that the Kriging model has already been constructed to correctly estimate the sign of the limit state function, equations (11) and (12) can be written as

$$
\begin{gathered}
\operatorname{Bel}(F)=\frac{1}{N} \sum_{k=1}^{N} I\left(\max _{x \in A_{k}} G(x)<0\right), \\
\operatorname{Pl}(F)=\frac{1}{N} \sum_{k=1}^{N} I\left(\min _{x \in A_{k}} G(x)<0\right) .
\end{gathered}
$$

To reduce the sample size, we use the expected risk function (ERF) $[23,39]$ as learning function to adaptively select the samples.

$$
\begin{aligned}
E[R(X)]= & -\operatorname{sign}(G(X)) G(X) \Phi\left(-\operatorname{sign}(G(X)) \frac{G(X)}{\widehat{s}(X)}\right) \\
& +\widehat{s}(X) \phi\left(\frac{G(X)}{\widehat{s}(X)}\right)
\end{aligned}
$$

where $\phi(\cdot)$ and $\Phi(\cdot)$ are the probability density function and $\mathrm{CDF}$ of standard normal distribution, respectively. The convergence criterion for the Kriging model is written as

$$
\frac{E\left[R\left(X^{*}\right)\right]}{(G(\bar{X})+\varepsilon)<10^{-4}}
$$

where $X^{*}$ is the value for maximum of ERF, $\bar{X}$ is the mean of the $X$, and $\varepsilon$ is $10^{-6}$.

3.3. Validation of Reliability Analysis with the Evidence Variables. The following numerical example is adopted to validate the proposed reliability analysis method. The limit state function is written as [13]

$$
g(x)=\frac{x_{1}^{2} x_{2}}{\alpha}-1,
$$

where $\alpha$ is a deterministic design variable and $x_{1}$ and $x_{2}$ are evidence variables. When $g<0$, the system is in the failure region. The BPA structures of $x_{1}$ and $x_{2}$ are listed in Table 1.

There are 13 points selected to construct the Kriging model. Then, $10^{5}$ Monte Carlo simulation is performed to calculate the $\mathrm{Pl}$ of the failure probability. The results of the reliability analysis are shown in Table 2. As can be seen, the result is close to the result from literature [13], which means the proposed evidence analysis method has a high accuracy.

3.4. Summary of EBMDO with Kriging-Based Reliability Analysis. First, a large population $S$ that contains $n_{\mathrm{MC}}$ points is generated. Points are randomly selected from population $S$. The limit state function is evaluated based on these points, 
TABLE 1: BPA structure of epistemic variables of validation case.

\begin{tabular}{|c|c|c|c|}
\hline \multicolumn{2}{|c|}{$x_{1}$} & \multicolumn{2}{|c|}{$x_{2}$} \\
\hline Interval & BPA (\%) & Interval & BPA (\%) \\
\hline$[3.0,4.0]$ & 2.2 & {$[50,64]$} & 2.2 \\
\hline$[4.0,4.5]$ & 13.6 & {$[64,72]$} & 13.6 \\
\hline$[4.5,4.75]$ & 15.0 & {$[72,76]$} & 15.0 \\
\hline$[4.75,5.0]$ & 19.2 & {$[76,80]$} & 19.2 \\
\hline$[5.0,5.25]$ & 19.2 & {$[80,84]$} & 19.2 \\
\hline$[5.25,5.5]$ & 15.0 & {$[84,88]$} & 15.0 \\
\hline$[5.5,6.0]$ & 13.6 & {$[88,96]$} & 13.6 \\
\hline$[6.0,7.0]$ & 2.2 & {$[96,106]$} & 2.2 \\
\hline
\end{tabular}

TABLE 2: Reliability analysis results for validation case.

\begin{tabular}{lcc}
\hline$\alpha$ & The proposed method & $\begin{array}{c}\text { Literature }[13] \\
\operatorname{Pl}(G)\end{array}$ \\
\hline 800 & $\operatorname{Pl}(G)$ & 1.0000 \\
900 & 1.0000 & 1.0000 \\
1000 & 1.0000 & 1.0000 \\
1100 & 1.0000 & 0.9995 \\
1200 & 0.9995 & 0.9890 \\
1300 & 0.9975 & 0.9860 \\
1400 & 0.9857 & 0.9818 \\
1500 & 0.9821 & 0.9102 \\
1600 & 0.9310 & 0.8313 \\
1700 & 0.8863 & 0.8047 \\
\hline
\end{tabular}

thus forming the initial DoE, $S_{\mathrm{DoE}}$, for the Kriging model. The Kriging model is then constructed, and the values of $E R F$ in population $S$ are calculated. The point with the largest value of ERF is selected and added to $S_{\mathrm{DoE}}$. The Kriging model is updated by $S_{\text {DoE }}$ until convergence is achieved.

Second, a reliability constraint is applied during optimization by the Kriging model. The framework of MDF and $\mathrm{CO}$ is adopted to achieve an optimal design.

The procedure of EBMDO is summarized as follows:

Step 1. Generate $n_{\mathrm{MC}}$ points $S$ according to the range of the variables

Step 2. Randomly select $n_{0}$ points from $S$, evaluate the limit state function, and form the initial $S_{\mathrm{DoE}}$

Step 3. Construct the Kriging model based on $S_{\text {DoE }}$

Step 4. Calculate the ERF on each point in $S$, and pick out point $x^{*}$ with the maximum value of ERF

Step 5. Evaluate the limit state function on the point $x^{*}$, and add the sample in $S_{\mathrm{DoE}}$

Step 6. If the Kriging model is converged, go to next step; otherwise, go to Step (3)

Step 7. Set the initial value of the optimization

Step 8. At each optimal point during optimization, evaluate the reliability of the system by the Kriging model

Step 9. If the reliability constraint is satisfied and the objective function is converged, stop; otherwise, go to Step (8)

During Kriging modeling, MDA should be performed to evaluate the limit state function. During optimization, MDA is not required for reliability analysis because the Kriging model is constructed to approximate the limit state function in the entire design space. Therefore, computational efficiency is considerably improved.

\section{Application Examples}

The proposed methodology is validated with two examples of EBMDO. For the inequality constraints of failure probability, evidence theory is used to estimate the epistemic uncertainty in terms of failure plausibility.

4.1. Case 1. The first EMBDO problem is a simple mathematical example. The problem is defined as

$$
\begin{aligned}
\text { find } & d=\left[d_{1}, d_{2}\right], \\
\min & f=d_{1}^{2}+d_{2}^{2}, \\
\text { s.t. } & \operatorname{Pl}\left[g_{i}(x)<0\right]<P_{\mathrm{f}}, \\
& g_{1}(x)=4-\beta x_{1}-x_{2}, \\
& g_{2}(x)=x_{1}+\beta x_{2}-2, \\
& 0 \leq d_{i} \leq 5, \\
& i=1,2, \beta=0.5,
\end{aligned}
$$

where $d_{1}, d_{2}$ are the design variables and $x_{1}, x_{2}$ are the evidence variables, and $x_{i}=d+\Delta x_{i}$. The system utilizes two design variables and outputs two states. They are not shared variables, thus the systems are uncoupled. The objective function is nonlinear with two constraints. $P_{\mathrm{f}}$ is the constraint of failure probability. The BPA structure of the uncertain variables is shown in Table 3, and the corresponding bar chart of epistemic variables is illustrated in Figure 4 .

The CO architecture of this problem can be written as one system optimization and two discipline optimizations. The system optimization is written as

$$
\begin{aligned}
\text { find } & d=\left[d_{1 \mathrm{sl}}, d_{2 \mathrm{sl}}\right], \\
\min & f=d_{1 \mathrm{ll}}^{2}+d_{2 \mathrm{sl}}^{2}, \\
\text { s.t. } & J_{1}=\left(d_{1 \mathrm{sl}}-d_{11}\right)^{2}+\left(d_{2 \mathrm{sl}}-d_{21}\right)^{2} \leq 10^{-5}, \\
& J_{2}=\left(d_{1 \mathrm{sl}}-d_{12}\right)^{2}+\left(d_{2 \mathrm{sl}}-d_{22}\right)^{2} \leq 10^{-5},
\end{aligned}
$$

where $d_{1 \mathrm{sl}}, d_{2 \mathrm{sl}}$ are the system-level design variables, $d_{i j}$ denotes the $i^{\text {th }}$ variables in the $j^{\text {th }}$ discipline, and $J_{1}, J_{2}$ are two auxiliary variables and the objective function of the discipline optimization problem. The auxiliary variables aim to reduce the difference of the system and discipline variables to $10^{-5}$. The optimization problem of discipline 1 is defined as

$$
\begin{aligned}
\text { find } & d=\left[d_{11}, d_{21}\right], \\
\min & J_{1}=\left(d_{1 \mathrm{sl}}-d_{11}\right)^{2}+\left(d_{2 \mathrm{sl}}-d_{21}\right)^{2}, \\
\text { s.t. } & \operatorname{Pl}\left(g_{1}(x)<0\right)<P_{\mathrm{f}}, \\
& g_{1}(x)=4-\beta x_{11}-x_{21} .
\end{aligned}
$$

The optimization problem of discipline 2 is defined as 
TABLE 3: BPA structure of epistemic variables of Case 1.

\begin{tabular}{lc}
\hline$x_{i}$ & BPA (\%) \\
\hline$\left[d_{i}-0.1, d_{i}+0.1\right]$ & 100 \\
{$\left[d_{i}-0.1, d_{i}-0.05\right]$} & 10 \\
{$\left[d_{i}-0.05, d_{i}\right]$} & 40 \\
{$\left[d_{i}, d_{i}+0.05\right]$} & 40 \\
{$\left[d_{i}+0.05, d_{i}+0.1\right]$} & 10 \\
\hline
\end{tabular}

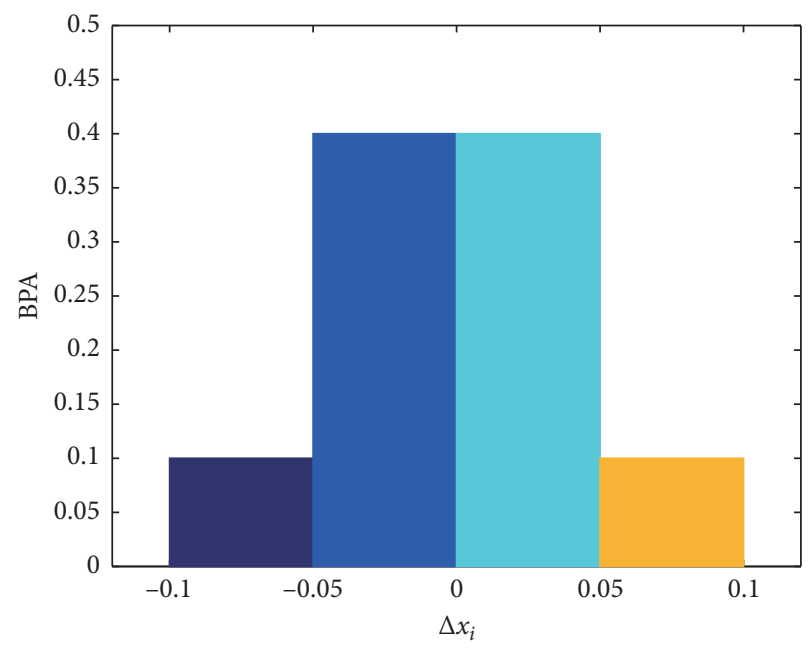

FIGURE 4: Illustration of BPA of epistemic variables.

$$
\begin{aligned}
\text { find } & d=\left[d_{12}, d_{22}\right], \\
\min & J_{2}=\left(d_{1 \mathrm{sl}}-d_{12}\right)^{2}+\left(d_{2 \mathrm{sl}}-d_{22}\right)^{2}, \\
\text { s.t. } & \operatorname{Pl}\left(g_{2}(x)<0\right)<P_{\mathrm{f}}, \\
& g_{1}(x)=x_{12}+\beta x_{22}-2 .
\end{aligned}
$$

The initial value of the design variables are set to [1.6, 0.8 ], which is the result of deterministic optimization (DO). To verify the accuracy of the proposed approach, MCS combined with the MDF architecture is used to directly solve EBMDO. The nonlinear sequential quadratic programming (NLPQL) is adopted as the optimization algorithm for both system and discipline optimization.

Firstly, the $10^{5}$ candidate points are generated by Latin hypercube sampling. Then, according to experience, 12 points are randomly selected and evaluated by executing the disciplinary analysis. The Kriging model is constructed and updated by the proposed active global learning method. Subsequently, the MDF and CO framework are performed based on the Kriging model to search the optimal solutions.

The results are summarized and compared with those of the deterministic design and MCS in Table 4. The second row shows the deterministic results. EBMDO is performed with three failure probability constraints, namely, $P_{\mathrm{f}}=$ $0.2,0.1,0.0013$. The results show that the objective function of MDO is smaller than that of EBMDO. The constraints of failure probability are not satisfied. For a small failure probability constraint, the design variables and the objective function must be large to meet the requirement of failure plausibility. The obtained results are close to those of MCS, and only the second constraint is active. The outer optimization loop has 100 iterations, and for each design point, $10^{5}$ times of MCS are performed in the evidence analysis. For each evidence analysis, the limit state function is called for about 10 times to obtain the minimum value. Hence, the limit state function is called for approximately $2 \times 10^{8}$ times. The number of limit state function calls by the proposed method is 26 times for this example, which is only $1.3 \times 10^{-7}$ of MCS.

4.2. Case 2. The second example contains two disciplines with three design and two coupled variables. The constraint function is nonlinear. The EBMDO problem is defined by

$$
\begin{aligned}
\text { find } & d=\left[d_{1}, d_{2}, d_{3}\right], \\
\min & f=d_{2}^{2}+d_{3}+y_{1}+e^{-y_{2}}, \\
\text { s.t. } & \operatorname{Pl}\left(g_{i}(x)<0\right)<P_{\mathrm{f}}, \quad i=1,2, \\
& g_{1}=y_{1}-3.16, \\
& g_{2}=24-y_{2}, \\
& y_{1}=x_{1}^{2}+x_{2}+x_{3}-0.2 y_{2}, \\
& y_{2}=\sqrt{y_{1}}+x_{1}+x_{3}, \\
& -8 \leq d_{1} \leq 8,2 \leq d_{2}, d_{3} \leq 8,
\end{aligned}
$$

where $d_{i}(i=1,2,3)$ are design variables, $y_{i}(i=1,2)$ are coupled variables, $x_{i}(i=1,2)$ are uncertainty variables, and $P_{\mathrm{f}}$ is the constraint of failure probability, $P_{\mathrm{f}}=0.01$. In this example, the coupled variables make the problem highly complex. Probability theory is a special case of evidence theory. To compare the EBMDO design with the RBMDO design, the BPA for each interval of the uncertain variables is considered to be similar to the area under the probability density function used in RBMDO, as shown in Figure 5.

In RBMDO, $x_{i}$ pertains to normally distributed random parameters with $x_{i} \sim N\left(d_{i}, 0.5^{2}\right)$. The BPA structure of the uncertain variables for EBMDO is shown in Table 5.

The samples are generated in the entire design space, namely, $[-8,8] \times[2,8] \times[2,8]$. Subsequently, 12 points are randomly selected to construct the initial DoE. Then, the learning strategy mentioned in Section 3 is utilized to select the points for DoE. In each selected point, the multidisciplinary analysis must be performed to obtain the response of the limit state function. The training history curve of the Kriging model is shown in Figure 6.

The Kriging model converges in 9 and 13 iterations for the first and second limit state function, respectively. To verify the accuracy of the Kriging model, the signs of $10^{5}$ samples that are randomly generated in the design space are compared with that of the true limit state function. The histogram of the sign is illustrated in Figure 7. The predicted signs have a good match with that of the true function. The number of signs that are incorrectly predicted is only four, while the relative error is $4 \times 10^{-5}$.

Then, the problem is solved using the CO strategy. The EBMDO problem can be decomposed into one system 
Table 4: Computational results of Case 1.

\begin{tabular}{lccccccc}
\hline Probability constraints & Methods & $d_{1}$ & $d_{2}$ & $\mathrm{Pl}_{1}$ & $\mathrm{Pl}_{2}$ & Objective & $N_{\text {call }}$ of PF \\
\hline- & DO & 1.600 & 0.800 & 0 & 0.6670 & 3.200 & - \\
$P_{\mathrm{f}}=0.2$ & EBMDO-MDF & 1.712 & 0.754 & 0 & 0.1299 & 3.4525 & 26 \\
& EBMDO-CO & 1.703 & 0.752 & 0 & 0.12876 & 3.4657 & 26 \\
& MCS & 1.700 & 0.750 & 0 & 0.1230 & 3.4525 & $2 \times 10^{8}$ \\
$P_{\mathrm{f}}=0.1$ & EBMDO-MDF & 1.710 & 0.803 & 0 & 0.05031 & 3.56891 & 26 \\
& EBMDO-CO & 1.706 & 0.804 & 0 & 0.05018 & 3.55685 & 26 \\
$P_{\mathrm{f}}=0.0013$ & MCS & 1.700 & 0.800 & 0 & 0.0502 & 3.530 & $2 \times 10^{8}$ \\
& EBMDO-MDF & 1.723 & 0.858 & 0 & 0 & 3.70489 & 26 \\
& EBMDO-CO & 1.726 & 0.851 & 0 & 0 & 3.70328 & 26 \\
& MCS & 1.725 & 0.850 & 0 & 0 & 3.69813 & $2 \times 10^{8}$ \\
\hline
\end{tabular}
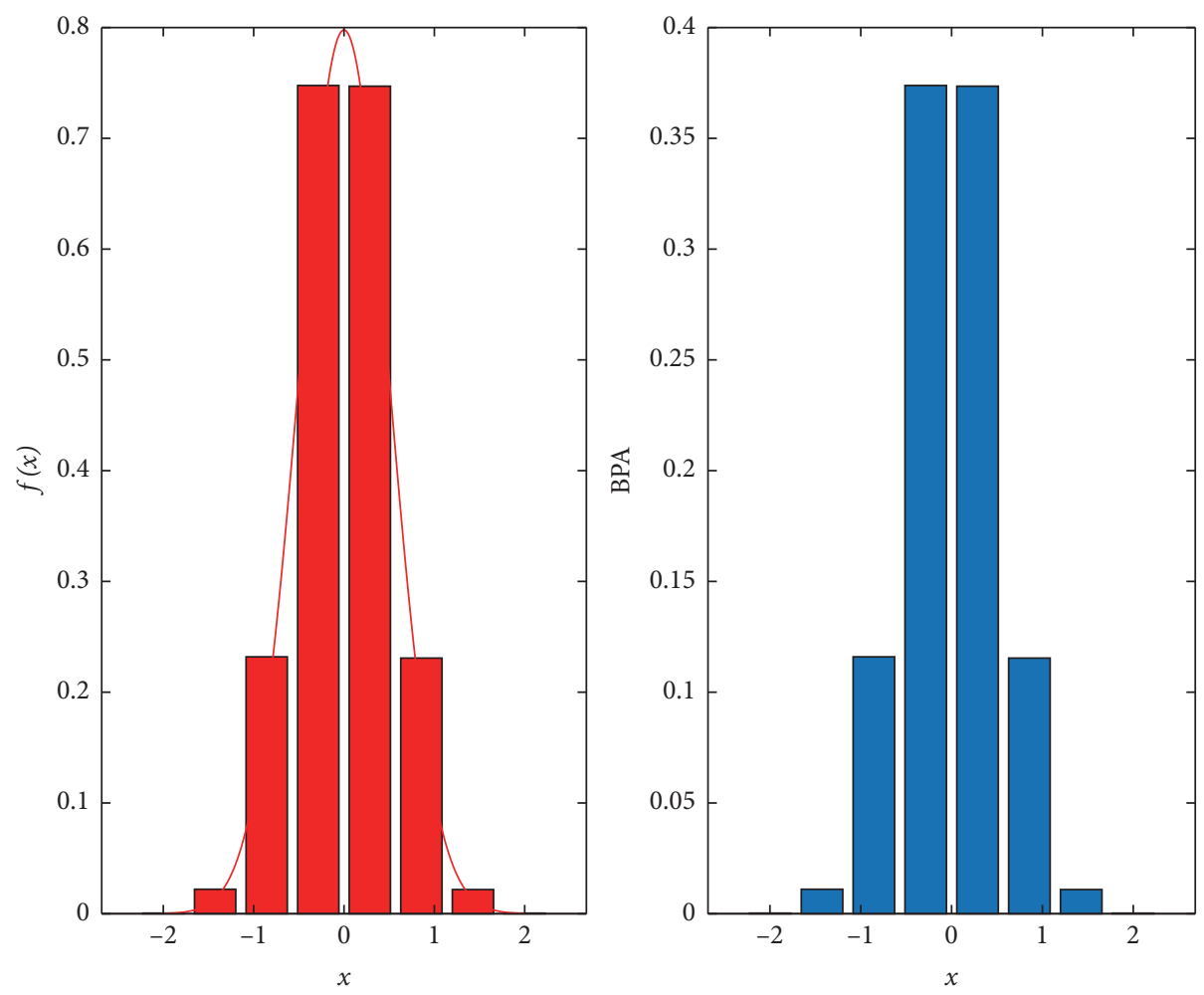

Figure 5: PDF and BPA for Case 2.

TABLE 5: BPA structure of epistemic variables of Case 2.

\begin{tabular}{lc}
\hline$x_{i}$ & $\mathrm{BPA}$ \\
\hline$\left[d_{i}-2, d_{i}+2\right]$ & 1 \\
{$\left[d_{i}-2.0, d_{i}-1.5\right]$} & 0.0014 \\
{$\left[d_{i}-1.5, d_{i}-1.0\right]$} & 0.0206 \\
{$\left[d_{i}-1.0, d_{i}-0.5\right]$} & 0.136 \\
{$\left[d_{i}-0.5, d_{i}\right]$} & 0.342 \\
{$\left[d_{i}, d_{i}+0.5\right]$} & 0.342 \\
{$\left[d_{i}+0.5, d_{i}+1.0\right]$} & 0.136 \\
{$\left[d_{i}+1.0, d_{i}+1.5\right]$} & 0.0206 \\
{$\left[d_{i}+1.5, d_{i}+2.0\right]$} & 0.0014 \\
\hline
\end{tabular}

problem and two corresponding discipline problems because of the presence of two disciplines. The system optimization problem is defined as

$$
\begin{array}{ll}
\text { find } & d=\left[d_{1 \mathrm{sl}}, d_{2 \mathrm{sl}}, d_{3 \mathrm{sl}}, y_{1 \mathrm{sl}}, y_{2 \mathrm{sl}}\right], \\
\min & f=d_{2 \mathrm{sl}}^{2}+d_{3 \mathrm{sl}}+y_{1 \mathrm{sl}}+e^{-y_{2 \mathrm{sl}},} \\
\text { s.t. } & J_{1}=\left(d_{11}-d_{1 \mathrm{SL}}\right)^{2}+\left(d_{21}-d_{2 \mathrm{SL}}\right)^{2}+\left(d_{31}-d_{3 \mathrm{SL}}\right)^{2} \\
& +\left(y_{11}-y_{1 \mathrm{SL}}\right)^{2} \leq 10^{-5}, \\
& J_{2}=\left(d_{12}-d_{1 \mathrm{SL}}\right)^{2}+\left(d_{32}-d_{3 \mathrm{SL}}\right)^{2}+\left(d_{22}-d_{2 \mathrm{SL}}\right)^{2} \leq 10^{-5},
\end{array}
$$

where $d_{i \mathrm{sl}}(i=1,2,3)$ contains the system design variables and $d_{1 i}, d_{2 i}, d_{3 i}$ denotes the design variables of the $i^{\text {th }}$ discipline. The coupled variables, $y_{1}, y_{2}$, are considered the design variables to decouple the disciplines.

The optimization problem of the first discipline is defined by 

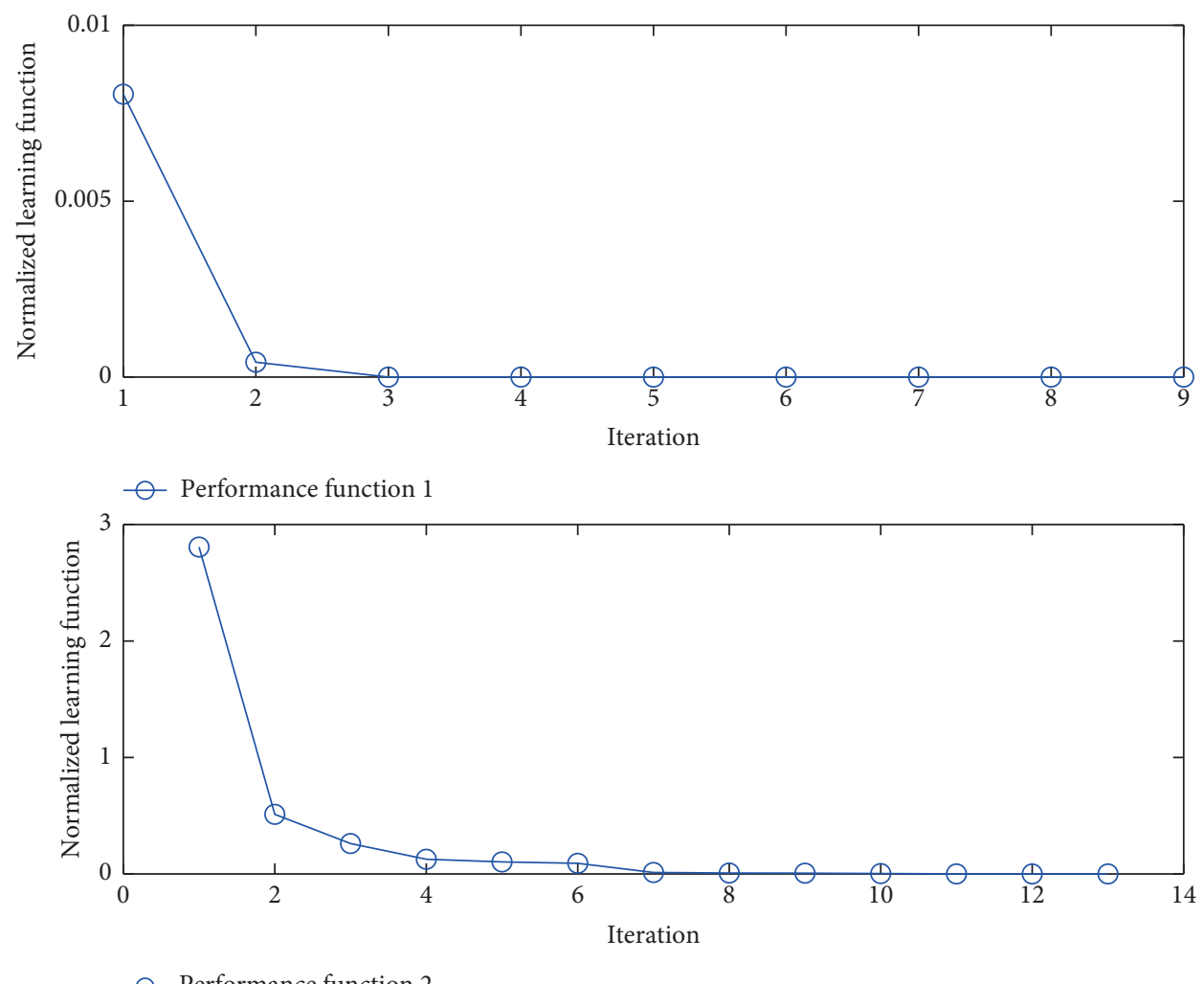

Figure 6: History of the training Kriging model.

$$
\begin{array}{ll}
\text { find } & d=\left[d_{11}, d_{21}, d_{31}, y_{11}, y_{21}\right], \\
\min & \left.J_{1}=\left(d_{11}-d_{1 \mathrm{sl}}\right)^{2}+\left(d_{21}-d_{2 \mathrm{sl}}\right)^{2}+\left(d_{31}-d_{3 \mathrm{sl}}\right)^{2}\right) \\
& \quad+\left(y_{11}-y_{1 \mathrm{sl}}\right)^{2}, \\
\text { s.t. } \quad & \operatorname{Pl}\left(g_{1}<0\right)<P_{\mathrm{f}}, \\
& g_{1}=y_{11}-3.16, \\
& y_{11}=x_{11}^{2}+x_{21}+x_{31}-0.2 y_{2 \mathrm{sl}} .
\end{array}
$$

The optimization problem of the second discipline is defined by

$$
\begin{aligned}
\text { find } & d=\left[d_{12}, d_{22}, d_{32}, y_{12}, y_{22}\right], \\
\min & J_{2}=\left(d_{12}-d_{1 \mathrm{sl}}\right)^{2}+\left(d_{32}-d_{3 \mathrm{sl}}\right)^{2}+\left(y_{22}-y_{2 \mathrm{sl}}\right)^{2}, \\
\text { s.t. } & \operatorname{Pl}\left(g_{2}<0\right)<P_{\mathrm{f}}, \\
& g_{2}=24-y_{22}, \\
& y_{22}=\sqrt{y_{1 \mathrm{l}}}+x_{12}+x_{32} .
\end{aligned}
$$

The data flowchart is presented in Figure 8 to show the solving architecture of cooperative optimization. The blue and red lines indicate the input and output, respectively. The system variables are passed to the discipline analysis, and the objective functions of disciplines are returned to system optimization. The objective functions of disciplines are the constraints of system optimization. The reliability constraints are evaluated by evidence analysis with the global Kriging models.

Table 6 compares the DO, EBMDO by MDF, EBMDO by $\mathrm{CO}, \mathrm{RBMDO}$, and MCS results. The conclusions are similar to those in the previous example. The evaluation number of the limit state function by the proposed approach is 46 , which is about $2.3 \times 10^{-7}$ that by MCS. This result indicates that the proposed method can ensure accuracy while having a great advantage over MCS.

4.3. Case 3. The third case is multidisciplinary design optimization for cooling blade which considers the aerodynamic, heat transfer, and strength analysis, revised from [40]. The geometry model of the cooling turbine blade and the design parameters are shown in Figure 9, where $x_{i}(i=1, \ldots, 4)$ is the thickness of ribs and $x_{i}(i=5, \ldots, 10)$ is the thickness of blade wall at different blade profile.

The EBMDO problem is defined by

$$
\begin{aligned}
\text { find } & \mathbf{x}=\left[x_{1}, \cdots, x_{10}\right], \\
\min & f=w_{1} D(\mathbf{x})+w_{2} T_{\mathrm{ave}}(\mathbf{x}), \\
\text { s.t. } & T_{\max } \leq 1500 \mathrm{~K}, \\
& \sigma_{\max } \leq 830 \mathrm{MPa}, \\
& P\{D-0.32 \geq 0\} \geq 99.9 \%, \\
& \mathbf{x}^{l} \leq \mathbf{x} \leq \mathbf{x}^{u},
\end{aligned}
$$




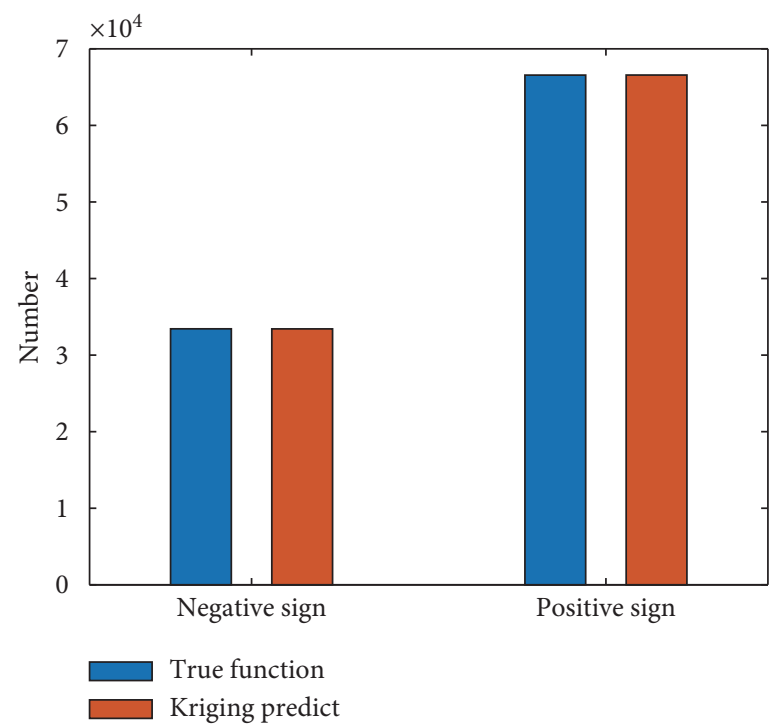

FIgURE 7: Accuracy validation of the global Kriging model.

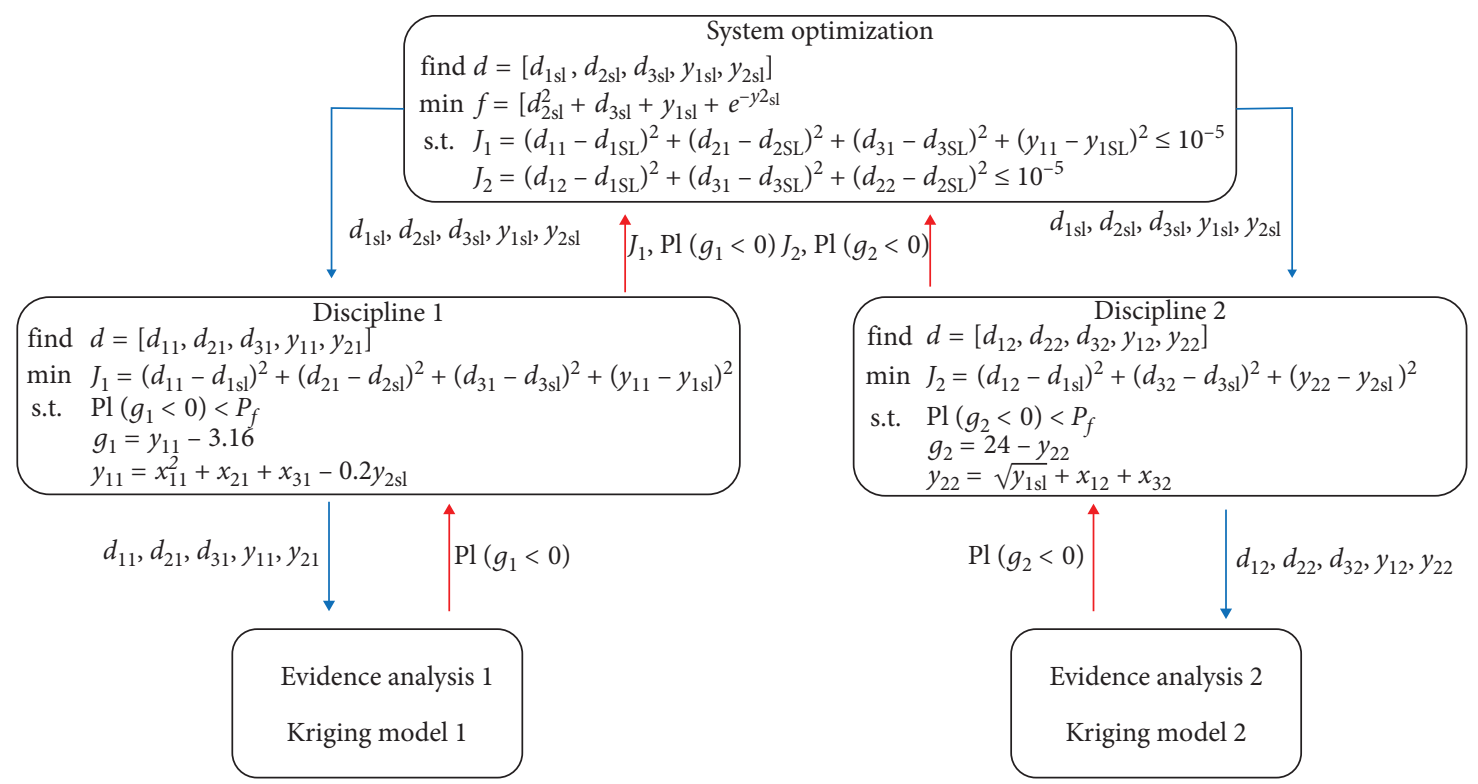

Figure 8: CO architecture of Case 2.

where $\mathbf{x}$ is the uncertainty design variables, $f$ is the objective function, and $D$ is the damage of cooling turbine blade, which is the function of the design variables. In this case, the damage $D$ is the whole damage, which is constituted by two parts, namely, the creep damage and the fatigue damage. The creep damage is predicted by the Larson-Miller equation [41] and the fatigue damage is calculated by the nominal stress method [42]. The fluid-thermal-solid interaction analysis is carried out to obtain the stress and the temperature in MDF framework. Also, in the CO framework, the three disciplines are decoupled and performed independently. $T_{\text {ave }}$ is the average temperature, $w_{1}=3000$ and $w_{2}=1$ are the weight factor, $T_{\max }$ is the maximum temperature of cooling turbine blade, $\sigma_{\max }$ is the maximum stress, $\mathbf{P}$ is the reliability, and $\mathbf{x}$ is the uncertainty design variables including $x_{1}, x_{2}, \ldots, x_{10}$. The design variables are considered as epistemic parameters. The lower and upper limit of variables are shown in Table 7. The thickness difference $\Delta x$ obeys threeparameter Weibull distribution, with the location parameter, the shape parameter, and the scale parameter as -0.1540 , 2.2979 , and 0.2923 , respectively. According to the Weibull distribution and Monte Carlo sampling, the BPA of the design variables are shown in Table 8.

The EBMDO results compared with the RBMDO and MDO by [40] are listed in Table 9. The multidisciplinary feasible approach is adopted in this case. As can be seen, the result obtained by EBMDO is very close to RBMDO, which indicates the proposed method is effective. 
TABle 6: Computational results of Case 2.

\begin{tabular}{|c|c|c|c|c|c|c|c|}
\hline Methods & $d_{1}$ & $d_{2}$ & $d_{3}$ & $\mathrm{Pl}_{1}$ & $\mathrm{Pl}_{2}$ & Objective & $N_{\text {call }}$ of $\mathrm{PF}$ \\
\hline $\mathrm{DO}$ & 0.11167 & 2.0 & 2.0 & 0.6125 & 0 & 9.2507 & 1 \\
\hline EBMDO-MDF & 1.87236 & 2.486 & 2.483 & 0.0093 & 0 & 15.7359 & 46 \\
\hline EBMDO-CO & 1.87562 & 2.483 & 2.482 & 0.0091 & 0 & 15.7275 & 46 \\
\hline RBMDO & 1.87167 & 2.0 & 2.24 & 0.00696 & 0 & 12.6556 & 46 \\
\hline MCS & 1.87167 & 2.480 & 2.480 & 0.0099 & 0 & 15.6927 & $2 \times 10^{8}$ \\
\hline
\end{tabular}
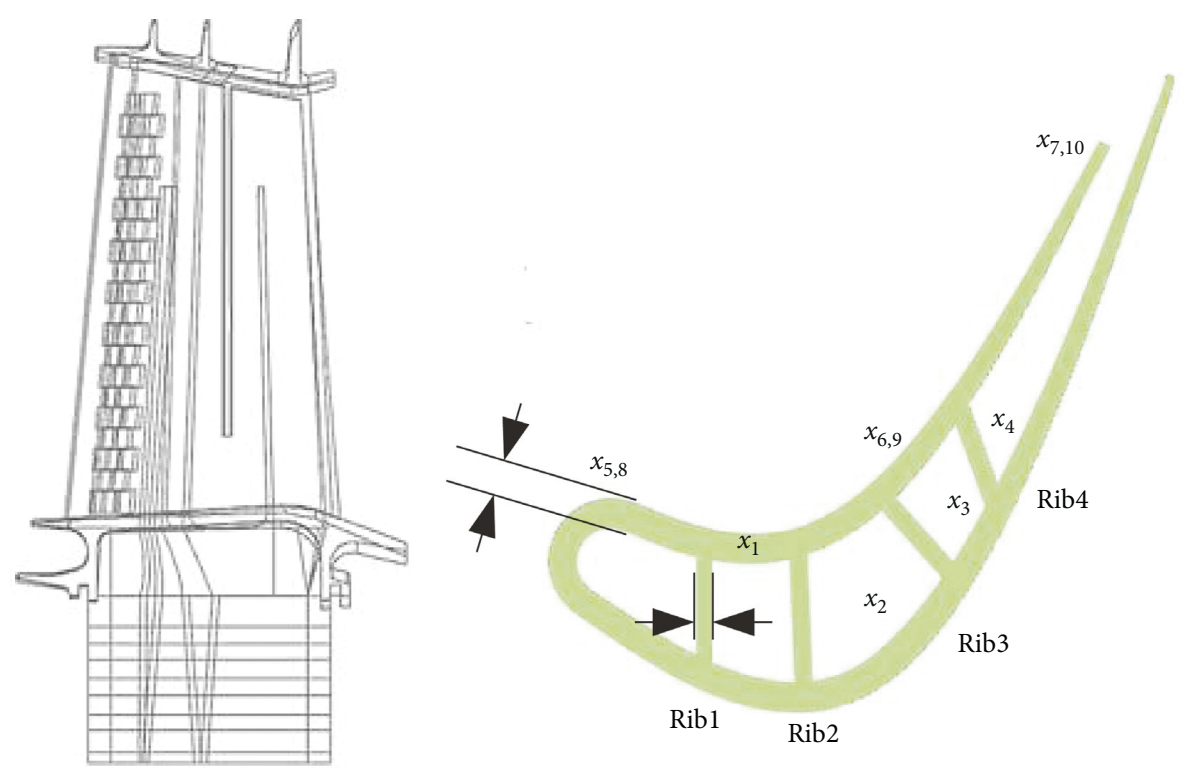

FIGURE 9: Geometry model and the design parameters of the blade [40].

TABLE 7: Lower and upper limit of variables.

\begin{tabular}{lccc}
\hline Design variables & & Original $(\mathrm{mm})$ & Boundary of variables \\
\hline Thickness of rib 1 & $x_{1}$ & 1.0 & {$[1.0,2.50]$} \\
Thickness of rib 2 & $x_{2}$ & 1.0 & {$[1.0,2.50]$} \\
Thickness of rib 3 & $x_{3}$ & 1.0 & {$[1.0,2.50]$} \\
Thickness of rib 4 & $x_{4}$ & 1.0 & {$[1.0,2.50]$} \\
Blade wall thickness at root & $x_{5}$ & 1.70 & {$[1.69,1.71]$} \\
of blade cross section & $x_{6}$ & 1.69 & {$[1.69,1.71]$} \\
& $x_{7}$ & 1.69 & {$[1.69,1.71]$} \\
Blade wall thickness at top & $x_{8}$ & 1.2 & {$[1.20,1.25]$} \\
of blade cross section & $x_{9}$ & 1.2 & {$[1.20,1.25]$} \\
& $x_{10}$ & 1.0 & {$[1.0,1.05]$} \\
\hline
\end{tabular}

TABLE 8: BPA structures of epistemic variables.

\begin{tabular}{lcc}
\hline Epistemic variables & Interval & BPA \\
\hline & {$[-0.15,0]$} & 0.1544 \\
Thickness difference $\Delta x$ & {$[0,0.15]$} & 0.4430 \\
& {$[0.15,0.30]$} & 0.3423 \\
& {$[0.30,0.45]$} & 0.0604 \\
\hline
\end{tabular}

\section{Conclusions}

This article presents a novel approach for EBMDO that combines the active global Kriging model, MDF, and CO. By
TABLE 9: Results of EBMDO and RBMDO [40].

\begin{tabular}{lcccc}
\hline Design variables & & MDO & RBMDO & EBMDO \\
\hline Thickness of rib 1 & $x_{1}$ & 2.50 & 2.4633 & 2.4814 \\
Thickness of rib 2 & $x_{2}$ & 2.0138 & 2.4316 & 2.4906 \\
Thickness of rib 3 & $x_{3}$ & 2.4275 & 2.2403 & 2.2127 \\
Thickness of rib 4 & $x_{4}$ & 2.4975 & 1.6709 & 1.6913 \\
Blade wall thickness at root of & $x_{5}$ & 1.70 & 1.7073 & 1.7032 \\
blade cross section & $x_{6}$ & 1.709 & 1.6939 & 1.6997 \\
& $x_{7}$ & 1.6917 & 1.7068 & 1.7085 \\
Blade wall thickness at top of & $x_{8}$ & 1.23 & 1.2276 & 1.2447 \\
blade cross section & $x_{9}$ & 1.2 & 1.2023 & 1.2458 \\
& $x_{10}$ & 1.05 & 1.0464 & 1.0965 \\
\hline
\end{tabular}


introducing a learning function, the global Kriging model is adaptively constructed to replace the limit state function. Then, the evidence analysis is performed by MCS and the Kriging model. The computational cost of EBMDO is reduced to approximately that of DO. Three examples are provided to illustrate the proposed approach. Compared with MCS, the proposed method can obtain accurate results and hold a significant advantage in terms of computational efficiency. Therefore, the proposed method is expected to be of great value in engineering applications.

As part of further work, some other architectures of multidisciplinary optimization can be taken into account in EBMDO, such as concurrent subspace optimization (CSSO) and bi-level integrated system synthesis (BLISS).

\section{Data Availability}

All relevant data used to support the findings of this study are included within the article.

\section{Conflicts of Interest}

The authors declare that they have no conflicts of interest.

\section{Acknowledgments}

This work has been supported by the Natural Science Foundation of Jiangsu Province (BK20190424), the Natural Science Foundation of Shaanxi Province (2019JQ-470, 2019JQ-609), the National Key R\&D Program of China (grant no. 2017YFB0202702), and the Center for High Performance Computing and System Simulation of Pilot National Laboratory for Marine Science and Technology (Qingdao).

\section{References}

[1] B. D. Youn, K. K. Choi, R.-J. Yang, and L. Gu, "Reliabilitybased design optimization for crashworthiness of vehicle side impact," Structural and Multidisciplinary Optimization, vol. 26, no. 3-4, pp. 272-283, 2004.

[2] T. W. Simpson, T. M. Mauery, J. Korte, and F. Mistree, "Kriging models for global approximation in simulationbased multidisciplinary design optimization," AIAA Journal, vol. 39, no. 12, pp. 2233-2241, 2001.

[3] F. Zhang, S. Tan, L. Zhang, Y. Wang, and Y. Gao, "Fault tree interval analysis of complex systems based on universal grey operation," Complexity, vol. 2019, Article ID 1046054, 8 pages, 2019.

[4] W. Yao, X. Chen, W. Luo, M. van Tooren, and J. Guo, "Review of uncertainty-based multidisciplinary design optimization methods for aerospace vehicles," Progress in Aerospace Sciences, vol. 47, no. 6, pp. 450-479, 2011.

[5] D. Meng, Y.-F. Li, H.-Z. Huang, Z. Wang, and Y. Liu, "Reliability-based multidisciplinary design optimization using subset simulation analysis and its application in the hydraulic transmission mechanism design," Journal of Mechanical Design, vol. 137, no. 5, Article ID 051402, 2015.

[6] J. C. Helton, "Uncertainty and sensitivity analysis in the presence of stochastic and subjective uncertainty," Journal of Statistical Computation and Simulation, vol. 57, no. 1-4, pp. 3-76, 1997.
[7] H. Agarwal, J. E. Renaud, E. L. Preston, and D. Padmanabhan, "Uncertainty quantification using evidence theory in multidisciplinary design optimization," Reliability Engineering \& System Safety, vol. 85, no. 1-3, pp. 281-294, 2004.

[8] C. Jiang, Z. Zhang, X. Han, and J. Liu, "A novel evidencetheory-based reliability analysis method for structures with epistemic uncertainty," Computers \& Structures, vol. 129, pp. 1-12, 2013.

[9] J. Zhou, Z. P. Mourelatos, and C. Ellis, "Design under uncertainty using a combination of evidence theory and a Bayesian approach," SAE International Journal of Materials and Manufacturing, vol. 1, no. 1, pp. 122-135, 2008.

[10] D. A. Alvarez, "On the calculation of the bounds of probability of events using infinite random sets," International Journal of Approximate Reasoning, vol. 43, no. 3, pp. 241-267, 2006.

[11] Z. Wang, H.-Z. Huang, Y. Li, Y. Pang, and N.-C. Xiao, “An approach to system reliability analysis with fuzzy random variables," Mechanism and Machine Theory, vol. 52, pp. 3546, 2012.

[12] S. Salehghaffari, M. Rais-Rohani, E. B. Marin, and D. J. Bammann, "Optimization of structures under material parameter uncertainty using evidence theory," Engineering Optimization, vol. 45, no. 9, pp. 1027-1041, 2013.

[13] M. Xiao, L. Gao, H. Xiong, and Z. Luo, "An efficient method for reliability analysis under epistemic uncertainty based on evidence theory and support vector regression," Journal of Engineering Design, vol. 26, no. 10-12, pp. 340-364, 2015.

[14] Z. Zhang, C. Jiang, G. G. Wang, and X. Han, "First and second order approximate reliability analysis methods using evidence theory," Reliability Engineering \& System Safety, vol. 137, pp. 40-49, 2015.

[15] R. Kang, Q. Zhang, Z. Zeng, E. Zio, and X. Li, "Measuring reliability under epistemic uncertainty: review on nonprobabilistic reliability metrics," Chinese Journal of Aeronautics, vol. 29, no. 3, pp. 571-579, 2016.

[16] J. Helton, J. D. Johnson, W. L. Oberkampf, and C. B. Storlie, "A sampling-based computational strategy for the representation of epistemic uncertainty in model predictions with evidence theory," Computer Methods in Applied Mechanics and Engineering, vol. 196, no. 37-40, pp. 3980-3998, 2007.

[17] V. Y. Kreinovich, A. Bernat, W. Borrett, Y. Mariscal, and E. Villa, Monte-Carlo Methods Make Dempster-Shafer Formalism Feasible, NASA Johnson Space Center, Houston, TX, USA, 1991.

[18] P. Limbourg and E. De Rocquigny, "Uncertainty analysis using evidence theory-confronting level-1 and level-2 approaches with data availability and computational constraints," Reliability Engineering \& System Safety, vol. 95, no. 5, pp. 550-564, 2010.

[19] N. Wilson, "The combination of belief: when and how fast?," International Journal of Approximate Reasoning, vol. 6, no. 3, pp. 377-388, 1992.

[20] C. Joslyn and V. Kreinovich, "Convergence properties of an interval probabilistic approach to system reliability estimation," International Journal of General Systems, vol. 34, no. 4, pp. 465-482, 2005.

[21] C. Wang and H. G. Matthies, "Evidence theory-based reliability optimization design using polynomial chaos expansion," Computer Methods in Applied Mechanics and Engineering, vol. 341, pp. 640-657, 2018.

[22] H.-R. Bae, R. V. Grandhi, and R. A. Canfield, "Uncertainty quantification of structural response using evidence theory," AIAA Journal, vol. 41, no. 10, pp. 2062-2068, 2003. 
[23] X. Yang, Y. Liu, and P. Ma, "Structural reliability analysis under evidence theory using the active learning Kriging model," Engineering Optimization, vol. 49, no. 11, pp. 19221938, 2017.

[24] Y. C. Bai, X. Han, C. Jiang, and J. Liu, "Comparative study of metamodeling techniques for reliability analysis using evidence theory," Advances in Engineering Software, vol. 53, pp. 61-71, 2012.

[25] Z. P. Mourelatos and J. Zhou, "A design optimization method using evidence theory," Journal of Mechanical Design, vol. 128, no. 4, pp. 901-908, 2006.

[26] Z. L. Huang, C. Jiang, Z. Zhang, T. Fang, and X. Han, “A decoupling approach for evidence-theory-based reliability design optimization," Structural and Multidisciplinary Optimization, vol. 56, no. 3, pp. 647-661, 2017.

[27] M. Xiao, H. Xiong, L. Gao, and Q. Yao, "An efficient method for structural reliability analysis using evidence theory," in Proceedings of the 2014 IEEE 17th International Conference on Computational Science and Engineering (CSE), December 2014.

[28] R. K. Srivastava and K. Deb, "An EA-based approach to design optimization using evidence theory," in Proceedings of the 13th Annual Conference on Genetic and Evolutionary Computation, July 2011.

[29] J. Zhang, M. Xiao, L. Gao, H. Qiu, and Z. Yang, "An improved two-stage framework of evidence-based design optimization," Structural and Multidisciplinary Optimization, vol. 58, no. 4, pp. 1673-1693, 2018.

[30] L. P. He and F. Z. Qu, "Possibility and evidence theory-based design optimization: an overview," Kybernetes, vol. 37, no. 9/ 10, pp. 1322-1330, 2008.

[31] W. Yao, X. Chen, Q. Ouyang, and M. van Tooren, "A reliability-based multidisciplinary design optimization procedure based on combined probability and evidence theory," Structural and Multidisciplinary Optimization, vol. 48, no. 2, pp. 339-354, 2013.

[32] X. Hu, X. Chen, G. T. Parks, and W. Yao, "Review of improved Monte Carlo methods in uncertainty-based design optimization for aerospace vehicles," Progress in Aerospace Sciences, vol. 86, pp. 20-27, 2016.

[33] K. Zaman and S. Mahadevan, "Reliability-based design optimization of multidisciplinary system under aleatory and epistemic uncertainty," Structural and Multidisciplinary Optimization, vol. 55, no. 2, pp. 681-699, 2017.

[34] C. Lin, F. Gao, and Y. Bai, "An intelligent sampling approach for metamodel-based multi-objective optimization with guidance of the adaptive weighted-sum method," Structural and Multidisciplinary Optimization, vol. 57, no. 3, pp. 10471060, 2018.

[35] B. Echard, N. Gayton, and M. Lemaire, "AK-MCS: an active learning reliability method combining Kriging and Monte Carlo simulation," Structural Safety, vol. 33, no. 2, pp. 145154, 2011.

[36] X. Du, A. Sudjianto, and B. Huang, "Reliability-based design with the mixture of random and interval variables," Journal of Mechanical Design, vol. 127, no. 6, pp. 1068-1076, 2005.

[37] F. Yang, Z. Yue, L. Li, and D. Guan, "Hybrid reliability-based multidisciplinary design optimization with random and interval variables," Proceedings of the Institution of Mechanical Engineers, Part O: Journal of Risk and Reliability, vol. 232, no. 1, pp. 52-64, 2018.

[38] A. Tavassoli, K. H. Hajikolaei, S. Sadeqi, G. G. Wang, and E. Kjeang, "Modification of DIRECT for high-dimensional design problems," Engineering Optimization, vol. 46, no. 6, pp. 810-823, 2014.

[39] X. Yang, Y. Liu, and Y. Gao, "Unified reliability analysis by active learning Kriging model combining with random-set based Monte Carlo simulation method," International Journal for Numerical Methods in Engineering, vol. 108, no. 11, pp. 1343-1361, 2016.

[40] L. Li, H. Wan, W. Gao, F. Tong, and H. Li, "Reliability based multidisciplinary design optimization of cooling turbine blade considering uncertainty data statistics," Structural and Multidisciplinary Optimization, vol. 59, no. 2, pp. 659-673, 2019.

[41] A. K. Gupta and M. R. Haider, "Creep life estimation of low pressure reaction turbine blade," International Journal of Technological Exploration and Learning (IJTEL), vol. 3, no. 2, pp. 402-404, 2014.

[42] H.-Z. Huang, C.-G. Huang, Z. Peng, Y.-F. Li, and H. Yin, "Fatigue life prediction of fan blade using nominal stress method and cumulative fatigue damage theory," International Journal of Turbo \& Jet-Engines, 2017. 


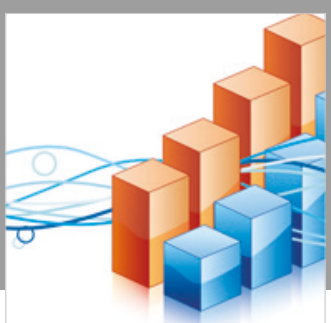

Advances in

Operations Research

\section{-n-m}
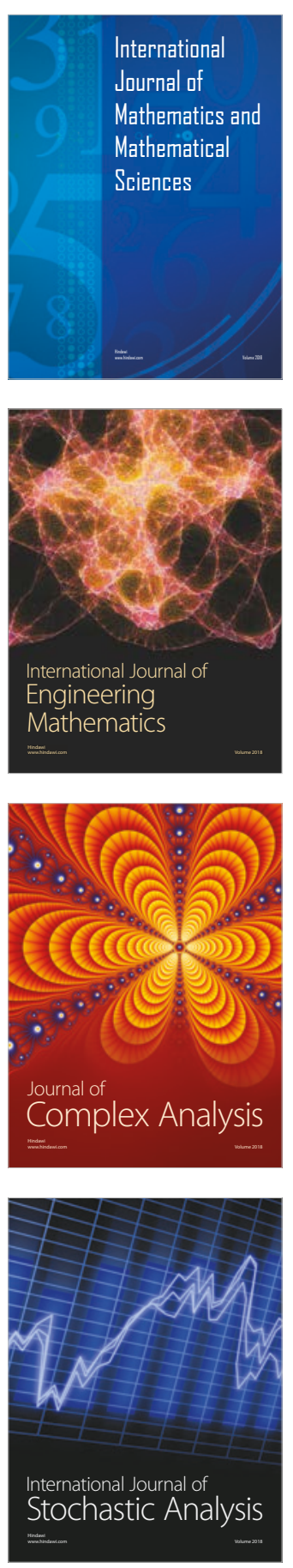
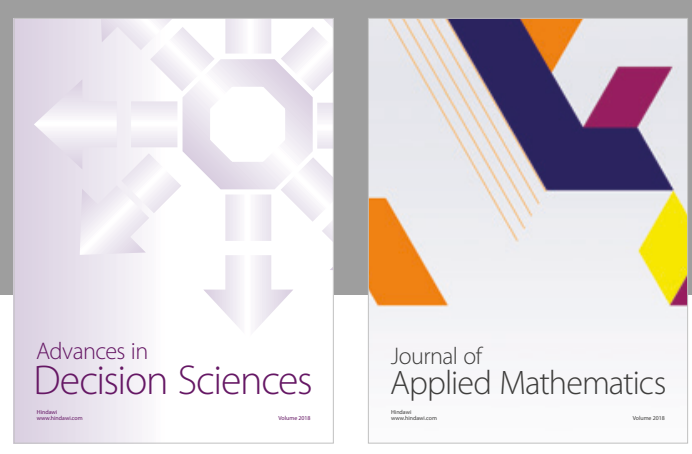

Journal of

Applied Mathematics
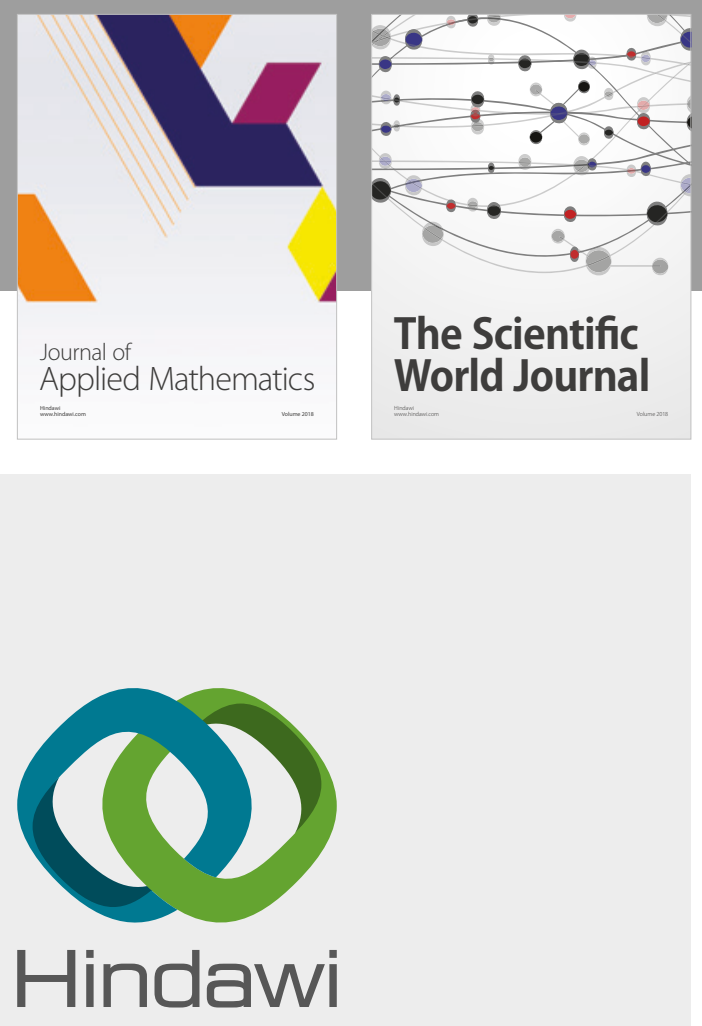

Submit your manuscripts at

www.hindawi.com

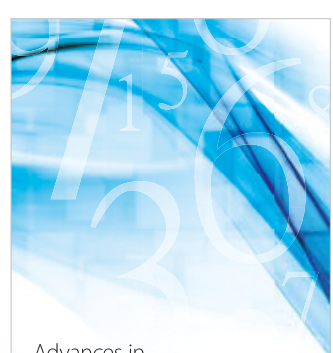

Advances in
Numerical Analysis
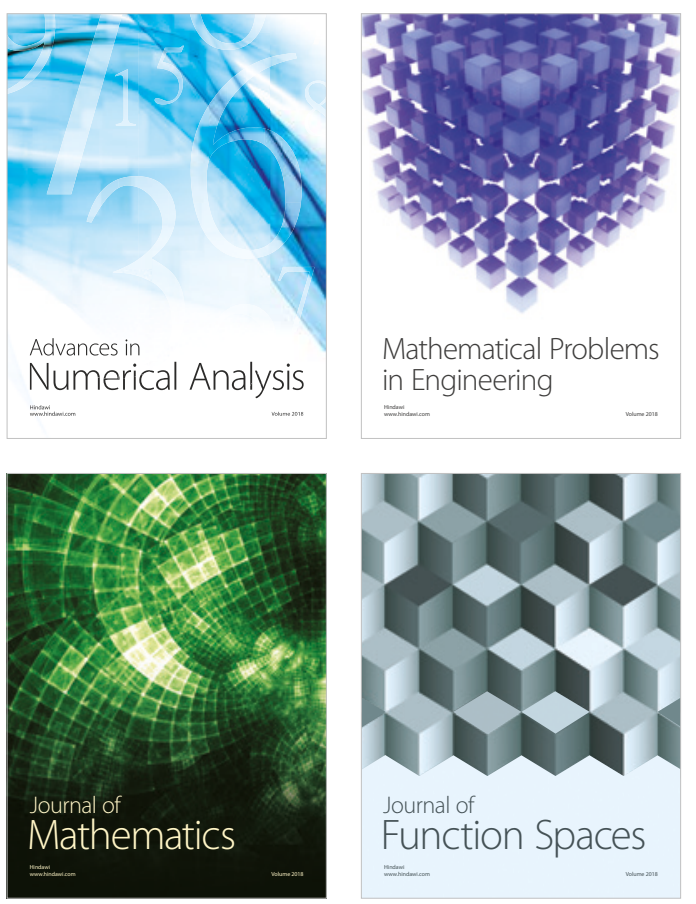

Mathematical Problems in Engineering

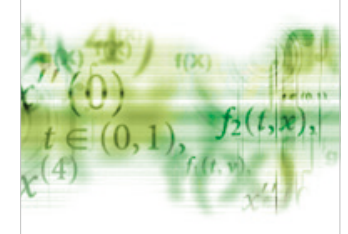

International Journal of

Differential Equations

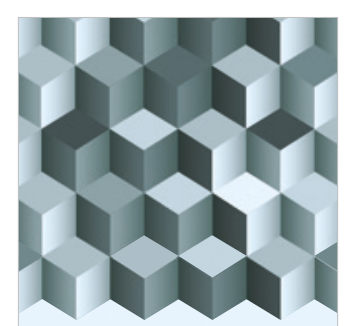

Journal of

Function Spaces
The Scientific

World Journal

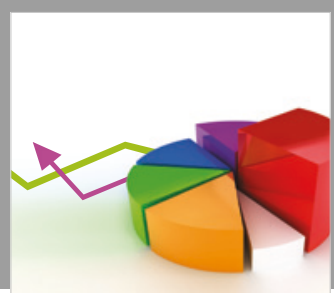

Journal of

Probability and Statistics
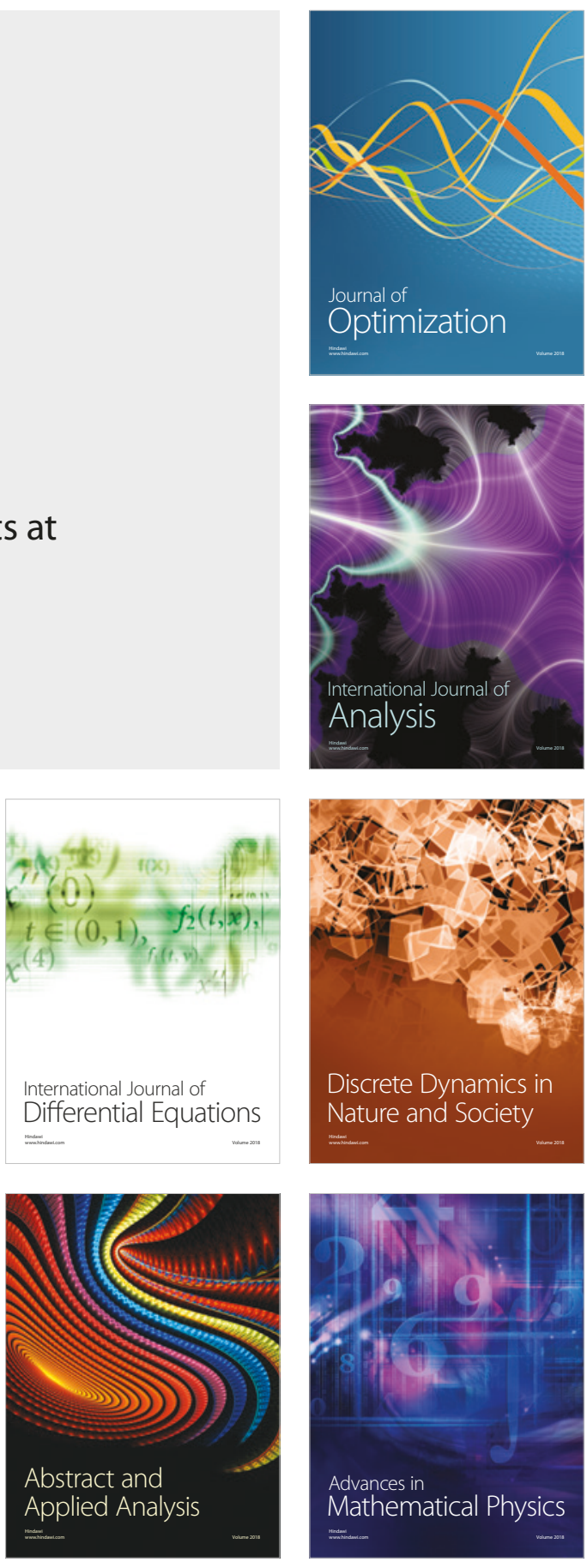